\title{
Long Noncoding RNA FAM201A Mediates the Radiosensitivity of Esophageal Squamous Cell Cancer by Regulating ATM and mTOR Expression via miR-101
}

OPEN ACCESS

Edited by:

Binhua Tang,

Hohai University, China

Reviewed by:

Shaoli Das,

National Institutes of Health (NIH), United States

Suman Ghosal,

National Institutes of Health (NIH),

United States

*Correspondence: Jiancheng Li

jianchengli6@126.com

Junqiang Chen

junqiangc@163.com

tThese authors have contributed equally to this work

Specialty section:

This article was submitted to Bioinformatics and Computational

Biology,

a section of the journal

Frontiers in Genetics

Received: 13 August 2018 Accepted: 19 November 2018 Published: 05 December 2018

Citation:

Chen M, Liu P, Chen Y, Chen Z

Shen M, LiUX, LiX, Li A, Lin Y, Yang $R$, Ni W, Zhou $X$, Zhang $L$, Tian Y, Li J and Chen J (2018) Long Noncoding RNA FAM201A Mediates the Radiosensitivity of Esophageal Squamous Cell Cancer by Regulating ATM and mTOR Expression via miR-101. Front. Genet. 9:611. doi: 10.3389/fgene.2018.00611

\begin{abstract}
Mingqiu Chen ${ }^{1,2,3 \dagger}$, Pingping Liu ${ }^{4+}$, Yuangui Chen ${ }^{5 \dagger}$, Zhiwei Chen ${ }^{6}$, Minmin Shen ${ }^{4}$, Xiaohong Liu ${ }^{4}$, Xiqing $\mathrm{Li}^{4}$, Anchuan $\mathrm{Li}^{5}$, Yu Lin ${ }^{7}$, Rongqiang Yang ${ }^{8}, \mathrm{Wei} \mathrm{Ni}^{8}$, Xin Zhou ${ }^{8}$, Lurong Zhang ${ }^{7}$, Ye Tian ${ }^{2,3}$, Jiancheng $\mathrm{Li}^{7 *}$ and Junqiang Chen ${ }^{7 *}$
\end{abstract}

\begin{abstract}
'Department of Radiation Oncology, Fujian Medical University Union Hospital and Fujian Provincial Platform for Medical Laboratory Research of First Affiliated Hospital, Fujian, China, ${ }^{2}$ Department of Radiation Oncology, The Second Affiliated Hospital of Soochow University, Suzhou, China, ${ }^{3}$ Institute of Radiotherapy \& Oncology, Soochow University, Suzhou, China, ${ }^{4}$ Shengli Clinical Medical College, Fujian Medical University, Fuzhou, China, ${ }^{5}$ Department of Radiation Oncology, Fujian Medical University Union Hospital, Fuzhou, China, ${ }^{6}$ Fuzhou Center for Disease Control and Prevention, Fuzhou, China, ${ }^{7}$ Department of Radiation Oncology, Fujian Cancer Hospital \& Fujian Medical University Cancer Hospital, Fuzhou, China, ${ }^{8}$ Cancer and Genetics Research Complex, Department Molecular Genetics and Microbiology, College Medicine, University of Florida, Gainesville, FL, United States
\end{abstract}

Background: The aim of the present study was to identify the potential long non-coding (Inc.)-RNA and its associated molecular mechanisms involved in the regulation of the radiosensitivity of esophageal squamous cell cancer (ESCC) in order to assess whether it could be a biomarker for the prediction of the response to radiotherapy and prognosis in patients with ESCC.

Methods: Microarrays and bioinformatics analysis were utilized to screen the potential IncRNAs associated with radiosensitivity in radiosensitive $(n=3)$ and radioresistant $(n=3)$ ESCC tumor tissues. Reverse transcription-quantitative polymerase chain reaction (RT-qPCR) was performed in 35 ESCC tumor tissues (20 radiosensitive and 15 radioresistant tissues, respectively) to validate the IncRNA that contributed the most to the radiosensitivity of ESCC (named the candidate InCRNA). MTT, flow cytometry, and western blot assays were conducted to assess the effect of the candidate IncRNA on radiosensitivity in vitro in ECA109/ECA109R ESCC cells. A mouse xenograft model was established to confirm the function of the candidate IncRNA in the radiosensitivity of ESCC in vivo. The putative downstream target genes regulated by the candidate IncRNA were predicted using Starbase 2.0 software and the TargetScan database. The interactions between the candidate IncRNA and the putative downstream target genes were examined by Luciferase reporter assay, and were confirmed by PCR.

Results: A total of 113 aberrantly expressed IncRNAs were identified by microarray analysis, of which family with sequence similarity 201-member A (FAM201A) was identified as the InCRNA that contributed the most to the radiosensitivity of ESCC. FAM201A was upregulated in radioresistant ESCC tumor tissues and had a poorer short-term response to radiotherapy resulting in inferior overall survival. FAM201A 
knockdown enhanced the radiosensitivity of ECA109/ECA109R cells by upregulating ataxia telangiectasia mutated (ATM) and mammalian target of rapamycin (mTOR) expression via the negative regulation of miR-101 expression. The mouse xenograft model demonstrated that FAM201A knockdown improved the radiosensitivity of ESCC.

Conclusion: The IncRNA FAM201A, which mediated the radiosensitivity of ESCC by regulating ATM and mTOR expression via miR-101 in the present study, may be a potential biomarker for predicting radiosensitivity and patient prognosis, and may be a therapeutic target for enhancing cancer radiosensitivity in ESCC.

Keywords: ATM, esophageal squamous cell carcinoma, FAM201A, long noncoding RNA, miR-101, mTOR, radiosensitivity

\section{INTRODUCTION}

Globally, esophageal cancer (EC) is one of the most common types of cancer, with the 7 th highest incidence rate and 6th greatest rate of cancer-associated death (Bray et al., 2018). Surgery still plays an important role in the treatment of EC (Pennathur et al., 2013; Rustgi and El-Serag, 2014). However, due to the patients' physiological conditions, the tumor location or the tumor stage, only $\sim 25 \%$ of newly diagnosed patients are suitable for surgery (Short et al., 2017). For patients with unresectable EC, radiotherapy (RT) combined with chemotherapy is considered to be the optimal treatment (Sasaki and Kato, 2016).

However, predominantly because of local failure (Lloyd and Chang, 2014; Versteijne et al., 2014) which has been associated with intrinsic and/or acquired radioresistance (Chen X. et al., 2017), the survival rate in EC patients following RT is as low as $10-30 \%$ after 5 years (Cooper et al., 1999; Gwynne et al., 2011). Therefore, how to predict the radiosensitivity and resensitize patients is imperative in patients with EC treated with RT. Unfortunately, as the molecular mechanism of radioresistance, which is known to involve DNA repair proteins (Zafar et al., 2010), cell signal pathways (Dumont and Bischoff, 2012), angiogenesis (Francescone et al., 2011), cancer stem cells (Moncharmont et al., 2012), and autophagy (Chaachouay et al., 2011), is intricate and has not been elucidated thoroughly, there

\footnotetext{
Abbreviations: ATM, ataxia telangiectasia mutated; AUC, area under the curve; CASC2, cancer susceptibility candidate 2; CR, complete response; CTV, clinical target volume; DLEU2, deleted in lymphocytic leukemia 2; DLX6-AS1, DLX6 antisense RNA 1; DNA, deoxyribonucleic acid; DNA-PKcs, DNA-dependent protein kinase catalytic subunit; ECOG, Eastern Cooperative Oncology Group; ESCC, esophageal squamous cell carcinoma; FAM201A, family with sequence similarity 201-member A; GTV, gross tumor volume; HRR, homologous recombination repair; IC, Induction chemotherapy; lncRNA, long non-coding RNA; MCF2L-AS1, MCF2L antisense RNA 1. mTOR, mammalian target of rapamycin; MTT, 3-(4,5)-dimethylthiahiazo(-z-y1)-3,5-diphenytetrazoliumromide; miR, microRNA; NHEJ, non-homologous end joining; OS, overall survival; PCR, polymerase chain reaction; PD, progression of disease; $\mathrm{PR}$, partial response; RT-qPCR, reverse transcription-quantitative polymerase chain reaction; RI-DSB, radiation-induced double-strand breaks; RNA, ribonucleic acid; ROC, receiver operating characteristic; RT, radiotherapy; SD, stable disease; sh-RNA, short hairpin RNA; siRNA, small interfering RNA; TP, platinum compound plus taxane.
}

are currently no accurate biomarkers to predict radioresistance or therapeutic targets to enhance the radiosensitivity of EC.

Long non-coding RNAs (lncRNAs) are a new class of nonprotein-coding transcripts that are longer than 200 nucleotides (Qi and Du, 2013). A number of previous studies have demonstrated that lncRNAs are important regulators of gene expression, that control both physiological and pathological processes in development and diseases such as cancer (Kung et al., 2013). Recent studies have reported that lncRNAs also function as regulators of tumor radiosensitivity and may serve as biomarkers for tumor response to RT (Spizzo et al., 2012; Yu et al., 2012). However, radiosensitivity-associated lncRNAs in esophageal squamous cell carcinoma (ESCC) are rarely reported (Tong et al., 2014; Zhang et al., 2015; Li et al., 2016; Zhou et al., 2016).

In the present study, we demonstrated that the lncRNA family with sequence similarity 201-member A (FAM201A) contributed the most to the radioresistance of ESCC. Furthermore, functional and mechanistic analyses revealed that FAM201A contributed to radioresistance by upregulating ataxia telangiectasia mutated (ATM) and mammalian target of rapamycin (mTOR) expression via actions as a miR-101 sponge. This study first established a FAM201A-miR-101-ATM/mTOR regulatory network in ESCC, revealing a promising therapeutic strategy for treating ESCC with radioresistance.

\section{MATERIALS AND METHODS}

\section{Patients and Tissue Specimens}

The present study was approved by the Fujian Medical University Union Hospital Institutional Review Board (No. 2014KY001). All of the patients signed informed consent prior to treatment, and all of the information was anonymized prior to its analysis. The pretreatment work-up and eligibility criteria, details of radiotherapy and chemotherapy, criteria for toxicity, and shortterm response, follow-up and the statistical analysis of survival were presented in our previous study (Chen M. Q. et al., 2017).

Between July 2015 and March 2017, a total of 41 patients with ESCC who received RT were recruited. Tissue specimens obtained during pretreatment with esophagogastroduodenoscopy were histopathologically examined by two independent pathologists and were snap 
frozen in liquid nitrogen and then stored at $-80^{\circ} \mathrm{C}$ until RNA extraction.

Tissue specimens were divided into a radiosensitive group $(n=23)$ and a radioresistant group $(n=18)$ based on shortterm response to RT. The short-term responses to RT were classified as a clinically complete response (CR), partial response $(\mathrm{PR})$, stable disease $(\mathrm{SD})$, or progressive disease (PD) according to the Japanese Classification of Esophageal Cancer guidelines (Japan Esophageal Society, 2017). Of these, the CR and PR were termed radiosensitive group and the $\mathrm{SD}$ and $\mathrm{PD}$ were termed radioresistant group in the current study.

\section{Microarray Screening and Bioinformatics Analysis}

Microarray profiling was performed using three radiosensitive ESCC tumor tissues and three radioresistant ESCC tumor tissues. RNA extraction and sequential microarray hybridization were conducted by Biotechnology Company (Shanghai, China), and the detected human genome transcripts were obtained by the Human IncRNA array V6.0 (4x180 K; Agilent Technologies, Inc., Santa Clara, CA, USA). Bioinformatics analysis was performed using GeneSpring Software to obtain differentially expressed lncRNAs correlated with ESCC radiosensitivity.

\section{Cell Lines and Culture}

The ESCC cell line Eca109 was obtained from Chinese Academy of Sciences (Beijing, China). The corresponding radioresistant cells (Eca109R) were established from the parental cell line Eca109 by stepwise X-ray irradiation at $30 \mathrm{~Gy}$ in three fractions (10 Gy per fraction) (Da et al., 2017). Cells were cultured in RPMI-1640 medium (HyClone; GE Healthcare Life Sciences, Logan, UT, USA) with $10 \%(\mathrm{v} / \mathrm{v})$ fetal bovine serum (Thermo Fisher Scientific, Inc., Waltham, MA, USA) and antibiotics (100 $\mathrm{U} / \mathrm{mL}$ penicillin and $100 \mu \mathrm{g} / \mathrm{mL}$ streptomycin; HyClone) in an atmosphere of $95 \%$ air $/ 5 \% \mathrm{CO}_{2}$ at $37^{\circ} \mathrm{C}$.

\section{RNA Isolation and Reverse Transcription-Quantitative Polymerase Chain Reaction (RT-qPCR)}

Total RNAs from either tissue samples or cultured cells were extracted with TRIzol reagent (Thermo Fisher Scientific, Inc.) according to the manufacturer's instructions. The RNA concentration and quality were measured using a NanoDrop ND2000 spectrophotometer which measured the absorbance at 260 and $280 \mathrm{~nm}$. Samples with an $\mathrm{A}_{260}: \mathrm{A}_{280}$ ratio $\geq 2.0$ were selected for further analysis.

First strand cDNA for the potential lncRNAs and putative micro (mi)-RNA were synthesized using the PrimeScript ${ }^{\mathrm{TM}}$ RT reagent kit with gDNA Eraser (Takara Biotechnology, Co., Ltd., Dalian, China) according to the manufacturer's protocol. Briefly, $1 \mu \mathrm{g}$ total RNA, $2 \mu 1$ 5X gDNA Eraser Buffer, $1 \mu \mathrm{lgDNA}$ Eraser and $\mathrm{RNase}$ Free $\mathrm{dH}_{2} \mathrm{O}$, were combined in a total reaction volume of $10 \mu \mathrm{l}$ and incubated at $42^{\circ} \mathrm{C}$ for $2 \mathrm{~min}$ to eliminate the genomic DNA. A total of $10 \mu \mathrm{l}$ of the RT reaction mixture (consisting of 4 $\mu 15 X$ PrimeScript Buffer 2, $1 \mu 1$ PrimeScript RT Enzyme Mix 1, 1 $\mu l$ RT Primer Mix, and $4 \mu$ l RNase Free $\mathrm{dH}_{2} \mathrm{O}$ ) was then added, and the mixture was incubated at $37^{\circ} \mathrm{C}$ for $15 \mathrm{~min}$, followed by $85^{\circ} \mathrm{C}$ for $5 \mathrm{~s}$ to generate the cDNA.

The expression of the potential lncRNAs in the radiosensitive tumor tissues, compared with the radioresistant tumor tissues, was quantified using SYBR ${ }^{\circledR}$ Premix Ex Taq (Takara Biotechnology Co., Ltd.) according to the manufacturer's instructions on the ABI 7500 Real-Time PCR System (Applied Biosystems; Thermo Fisher Scientific, Inc.). Briefly, the $20 \mu \mathrm{l}$ reaction mixtures were incubated at $95^{\circ} \mathrm{C}$ for $30 \mathrm{~s}$ for the initial denaturation, followed by 40 cycles at $95^{\circ} \mathrm{C}$ for $5 \mathrm{~s}$ and $60^{\circ} \mathrm{C}$ for $34 \mathrm{~s}$. The expression levels of lncRNAs were calculated using the $\Delta \mathrm{Ct}$ method, where $\Delta \mathrm{Ct}=\mathrm{Ct}$ target $-\mathrm{Ct}_{\text {reference, }}$ a smaller $\Delta \mathrm{Ct}$ value indicates a greater expression. The relative expression of lncRNAs was analyzed using the $2^{-\Delta \Delta C t}$ method (Livak and Schmittgen, 2001); data was normalized to the endogenous control GAPDH. Each sample was examined in triplicate. The primers and oligonucleotides of the plasmid were synthesized by Invitrogen (Thermo Fisher Scientific, Inc.), the sequences are presented in Table 1. The aberrant lncRNA that had the greatest sensitivity and specificity for predicting ESCC radiosensitivity (in radiosensitive and radioresistant tissues), as identified by receiver operating characteristic (ROC) curves, and was associated with survival, was identified as the candidate lncRNA for further study.

\section{Transient Transfection}

Small interfering RNA (siRNA) specifically targeting candidate lncRNA (si-candidate-lncRNA) and putative-miRNA, negative control (NC) si-candidate-lncRNA and si-putative-miRNA, candidate-lncRNA mimic, putative-miRNA mimic, and the inhibitor control were constructed by Nanjing Dongji Biotechnology Company (Nanjing, China). Ectopic expression of the candidate lncRNA was achieved by introducing the candidate lncRNA sequence into a pcDNA3.1 vector (Thermo Fisher Scientific, Inc.). Eca109/Eca109R cells were seeded into 6-well plates at a density of $1 \times 10^{6}$ cells/well and cultured overnight prior to transfection. Then, transient transfection with oligonucleotides or plasmids into Eca109/Eca109R cells was performed using Lipofectamine $2000^{\mathrm{TM}}$ (Thermo Fisher Scientific, Inc.). Cells were harvested $48 \mathrm{~h}$ post-transfection for subsequent analysis. PCR was used to validate the efficacy of Eca109/Eca109R cell transfection with si-candidate-lncRNA and candidate-lncRNA-mimic.

\section{Western Blot Analysis}

Protein samples from tissues or cells were subjected to $10 \%$ SDSPAGE and transferred to PVDF membranes. Following blocking in $5 \%$ skim milk for $2 \mathrm{~h}$, the membranes were incubated overnight at $4{ }^{\circ} \mathrm{C}$ with the primary antibodies against P-glycoprotein (Pgp; 1:1,000), glutathione S-transferase $\pi$ (GST- $\pi ; 1: 500)$, ATM (1:750), mTOR $(1: 1,000)$, and $\beta$-actin $(1: 5,000)$ purchased from Zen Bioscience Biotechnology, Inc. (Chengdu, China), followed by incubation with horseradish peroxidase-conjugated goat antirabbit secondary antibodies for $2 \mathrm{~h}(1: 5,000)$. The antigenantibody complexes were visualized using chemiluminescence. 
TABLE 1 | The primer sequences used in reverse transcription-quantitative polymerase chain reaction.

\begin{tabular}{|c|c|c|}
\hline Primers used for RT-qPCR & Forward $\left(5^{\prime}-3^{\prime}\right)$ & Reverse $\left(3^{\prime}-5^{\prime}\right)$ \\
\hline FAM201A & TCTCTGATGGGAGCCTCTITA & CAAGCCACAGACGGAGAAA \\
\hline CASC2 & GTCCGCATGGTAAGGAATCA & GACTGCGTITATCAAGTCCAAAG \\
\hline DLEU2 & TGGCGCAGTCGGTTAAT & TTCCTTGCAGTACACCTITCA \\
\hline DLX6-AS1 & TCTCCTCCTACCTAGCATCTTC & CCTITGAAGCTCCTACTCCTाT \\
\hline MCF2L-AS1 & TTGAGCCTGGGCAATGTAG & CTTCCTGCTGGAATTCTCTCTC \\
\hline GAPDH & CAGGGCTGCTITAACTCTGGTAA & GGGTGGAATCATATTGGAACATGT \\
\hline FAM201A mimic & GGGGTACCGAGTGCACCTGGCCTGAGAG & GGAAGCCTITGTGGTTAGATATITGAAAT \\
\hline \multicolumn{3}{|c|}{ OLIGONUCLEOTIDES OF PLASMID } \\
\hline SiFAM201A & GATCTITCGTCCATTTACTtt & \\
\hline NC-siFAM201A & GCCTTATTTCTATCTTACGtt & \\
\hline FAM201A-cDNA & $\begin{array}{l}\text { GTACCTCGATCПTCGTCCATIACПTCAAGAGA } \\
\text { GTAAATGGACGAAAGATCTITGGAAA }\end{array}$ & $\begin{array}{l}\text { AGCTITCCAAAAAGATCTTCGTCCATTACTCT } \\
\text { CTTGAAGTAAATGGACGAAAGATCGAG }\end{array}$ \\
\hline NC-FAM201A-cDNA & $\begin{array}{l}\text { GTACCTCGCCTTATTCTATCTTACGTCAAGAGC } \\
\text { GTAAGATAGAAATAAGGCTITGGAAA }\end{array}$ & $\begin{array}{l}\text { AGCTITCCAAAAAGCCTTATTCTATCTTACGCT } \\
\text { CTTGACGTAAGATAGAAATAAGGCGAG }\end{array}$ \\
\hline miR-101 & AAGUCAAUAGUGUCAUGACAU & \\
\hline miR-590 & GACGUGAAAAUACUUAUUCGAG & \\
\hline Negative control & UUCUCCGAACGUGUCACGUUU & \\
\hline
\end{tabular}

\section{Radiosensitivity Assay}

Radiosensitivity was assessed by 4-5-dimethylthiazol-2-yl)-2,5diphenyl tetrazolium bromide (MTT) assay. ECA109/ECA109R cells $(5,000 /$ well $)$ were incubated for $48 \mathrm{~h}$ prior to exposure to various doses of radiation $(0 \mathrm{~Gy}, 2 \mathrm{~Gy}, 4 \mathrm{~Gy}, 6 \mathrm{~Gy}, 8 \mathrm{~Gy}$, and $10 \mathrm{~Gy}$ ). Subsequently, $10 \mu \mathrm{l}$ of $5 \mathrm{mg} / \mathrm{mL}$ MTT was added to each well for a further $3 \mathrm{~h}$, followed by the addition of $150 \mu \mathrm{l}$ DMSO to dissolve the generated formazan crystals. The absorbance at a wavelength of $570 \mathrm{~nm}$ was detected using a microplate reader.

\section{Flow Cytometry Analysis of Apoptosis}

ECA109/ECA109R cells (5,000/well) were incubated for $48 \mathrm{~h}$ prior to exposure to various doses of radiation $(0 \mathrm{~Gy}, 2 \mathrm{~Gy}$, $4 \mathrm{~Gy}, 6 \mathrm{~Gy}, 8 \mathrm{~Gy}$, and $10 \mathrm{~Gy}$ ). The ratio of apoptotic cells was detected using an Annexin V-FITC Apoptosis Detection Kit (BD Bioscience, Franklin Lakes, NJ, USA) and analyzed using a BD Calibur flow cytometer with CellQuest software (BD Biosciences).

\section{Candidate IncRNA Downstream Target Genes and Luciferase Reporter Assay}

The potential target genes downstream of the candidate lncRNA were predicted using Starbase 2.0 software (http://starbase. sysu.edu.cn/starbase2/index.php) and the TargetScan (www. targetscan.org/vert_71/) database.

The full fragments of the candidate lncRNA or its mutant containing the putative miRNA-binding sites were synthesized and cloned downstream of the firefly luciferase gene in pGL3 plasmids (Promega Corporation, Madison, WI, USA), and were termed the pGL3-candidate IncRNA-wild type (Wt) and pGL3candidate lncRNA-mutant (Mut). Eca109 and Eca109R cells were maintained in 96-well plates and co-transfected with 400 ng of the constructed luciferase reporter plasmids, $50 \mathrm{ng}$ of
Renilla luciferase reporter vector and $50 \mathrm{nM}$ of the putative miRNA mimic, miR-con, or putative miRNA-vector using Lipofectamine $3000^{\mathrm{TM}}$ (Thermo Fisher Scientific, Inc.). Cells were harvested at $48 \mathrm{~h}$ after transfection, and luciferase activity was determined using a Dual Luciferase Reporter Assay Kit (Promega Corporation). Renilla luciferase activities were used as the internal control for the normalization of firefly luciferase activity.

\section{In vivo Experiments}

The animal experiments were approved by the Animal Care and Use Committee of Fujian Medical University Union Hospital and were performed in accordance with the Institutional Guide for the Care And Use Of Laboratory Animals. Lentiviral vector [Lenti-short hairpin (sh)-candidate lncRNA] for stable silenced expression of the candidate IncRNA was obtained from Shanghai GenePharma Co., Ltd. (Shanghai, China) and transfected into Eca109/Eca109R cells. The success of transfection was detected by PCR and the survival of the cells was determined by an MTT assay. Then, equal numbers of siRNA-candidate lncRNAtransfected Eca109, NC and control cells were implanted into 8 -week old nude mice $(n=5$ per group; Model Animal Research Center of Nanjing University) by subcutaneous injection.

At two weeks after the injection (to allow for tumor growth), the tumors were irradiated by X-ray at $10 \mathrm{~Gy}$. Tumor size was measured every 3 days with a caliper, and tumor volume was calculated according to the following formula: Volume $=$ (length $\mathrm{x}$ width $\left.{ }^{2}\right) / 2$. All mice were sacrificed on day 42 after inoculation. The resected tumor masses were harvested for subsequent weight measurements. A growth curve was constructed to determine tumor radiosensitivity and the effect of the siRNA of the candidate lncRNA on tumorigenicity in nude mice was analyzed. 


\section{Statistical Analysis}

The overall survival data was analyzed using SPSS software 23.0 (IBM Corp., Armonk, NY, USA). Survival curves were established through the Kaplan-Meier method and compared by a log rank test.

A multivariable analysis of patient demographic and clinical parameters (gender, age, ECOG score, tumor location, clinical $\mathrm{T}$ and $\mathrm{N}$ stages, the radiotherapy doses for GTV and CTV, and the tumor response to treatment) was performed using the Cox proportional hazards model.

Experimental data are presented as $\overline{\mathrm{x}} \pm \mathrm{s}$ from independent experiments performed in triplicate. For comparisons, paired or independent Student's $t$-tests, Chi-square tests or ANOVA with post hoc tests (Tukey's) were performed. ROC curves were used for selecting an optimal cut-off point for each test and for comparing the accuracy of diagnostic tests. Two-tailed $P<0.05$ $\left({ }^{*} P<0.05,{ }^{* *} P<0.01\right.$, $\left.{ }^{* * *} P<0.001\right)$ was considered to indicate a statistically significant difference.

\section{RESULTS}

\section{Patient Characteristics, Treatment Response, and Survival}

Between July 2015 and March 2017, a total of 41 ESCC patients treated with RT combined with chemotherapy were enrolled in the present study. After RT, a total of 4 patients achieved CR, 19 patients reached PR, 9 patients maintained SD and 9 cases had $\mathrm{PD}$. There were no significant differences between radiosensitive (4 CR and $19 \mathrm{PR}$ ) and radioresistant (9 SD and $9 \mathrm{PD}$ ) patients regarding the distributions of gender, age, ECOG score, tumor location, and clinical stage (Table 2).

\section{Differential Expression of IncRNAs Potentially Correlated With Radiosensitivity} A total of 113 aberrantly expressed lncRNAs were identified in the microarray analysis using three radiosensitive ESCC tumor tissues and three radioresistant ESCC tumor tissues, of which 71 lncRNA transcripts were upregulated (fold change $>2, P$ $<0.05$ ) and 42 lncRNA transcripts were downregulated (fold change $<0.5, P<0.05$ ) in the radiosensitive ESCC tumor tissues when compared with the radioresistant ESCC tumor tissues. The IncRNAs CASC2, FAM201A, DLEU2, DLX6-AS1, and MCF2LAS1 were considered to be the potential lncRNAs related to radiosensitivity when analyzed using GeneSpring Software 12.6 (Agilent Technologies, Inc.) (Figure 1A, Supplementary File 1).

Tumor tissues from the remaining 35 enrolled patients (20 radiosensitive patients and 15 radioresistant patients, respectively) were collected to detect the expression of the lncRNAs CASC2, FAM201A, DLEU2, DLX6-AS1, and MCF2LAS1 by RT-qPCR. The results revealed that the differential expression of CASC2, FAM201A, and DLX6-AS1 between the radioresistant and radiosensitive groups were significantly different, while the difference in the DLEU2 and MCF2L-AS1 expressions were not significantly different when comparing the groups (Figure 1B; Supplementary File 2).

\section{FAM201A Is a Novel IncRNA With a Potential Function in the Radiosensitivity and Survival of ESCC}

Based on above data, the ROC curve of the lncRNAs CASC2, FAM201A, and DLX6-AS1 was applied to identify the lncRNA that was the most correlated to radiosensitivity and survival using the area under curve (AUC) were 0.783 (95\%CI: 0.609 957, $P=0.005$ ), 0.817 (95\%CI: 0.673-960, $P=0.002$ ), and 0.340 (95\%CI: $0.150-530, P=0.110$ ); respectively. Compared with the lncRNA DLX6-AS1, FAM201A, and CASC2 yielded a superior AUC with specificity and sensitivity for distinguishing radiosensitive ESCC tumor tissues from radioresistant ESCC tumor tissues (Figure 2A).

CASC2 was associated with short-term response to RT but not with survival, while FAM201A was correlated with both the short-term response and survival (Figures 2B,C). This indicated that FAM201A, as opposed to CASC2, may be a suitable biomarker of ESCC treated with RT.

To analyze whether FAM201A functions as a biomarker for radiosensitivity and survival in ESCC or not, the maximum Youden index method (Fluss et al., 2005) was performed to establish the cutoff value of FAM201A in the ROC curve. A total of 22 patients were termed as FAM201A-low with an average $\Delta \mathrm{Ct}$ expression value of 6.155 , whereas, the remaining 13 patients, named the FAM201A-high expression group, had an average $\Delta \mathrm{Ct}$ expression value of 8.437 (Supplementary File 3).

Compared with the FAM201A-low group, the FAM201A-high group exhibited a poorer short-term response to RT and lower survival time. However, neither high or low FAM201A expression was correlated with tumor stage, regardless of whether it was $\mathrm{T}$ or $\mathrm{N}$ stage (Table 3). Furthermore, univariate and multivariate analysis indicated that FAM201A was the only independent risk factor for survival (OR, 0.642; 95\% CI, 0.4668-0.885; $P=0.007$ ). These data suggested that FAM201A could be a robust molecular marker for predicting RT sensitivity and survival in patients with ESCC.

\section{FAM201A Regulated Radiosensitivity in vitro}

Based on the above results, the effects of FAM201A regulated radiosensitivity in ESCC cancer cells were further explored by performing an X-ray irradiation experiment using Eca109/Eca109R cells transfected with si-FAM201A and FAM201A-mimic (Figures 3A,B; Supplementary File 4).

The results revealed that the survival rates of both Eca109 and Eca109R cells decreased with the increasing X-ray irradiation dose, and the percentage of apoptotic cells in each line increased with the increasing X-ray irradiation dose (Figures 3C,D; Supplementary File 4). The decrease in survival was more pronounced with the increase in X-ray irradiation dose in ECA109 cells when compared with ECA109R cells, demonstrating that the Eca109R cells were more resistant to X-ray irradiation.

In Eca109 cells, when compared with the control cells, FAM201A-mimic exhibited a significant promotion in cell proliferation, while si-FAM201A exhibited a significant 
TABLE 2 | Clinicopathological characteristics of the entire cohort of 41 patients with ESCC.

\begin{tabular}{|c|c|c|c|c|}
\hline Characters & Radiosensitive & Radioresistant & Total & $p$ \\
\hline Gender & & & & 0.706 \\
\hline Male & 17 & 15 & 32 & \\
\hline Female & 6 & 3 & 9 & \\
\hline Age (range) & $61(47-70)$ & $63(47-70)$ & $61(47-70)$ & 0.406 \\
\hline ECOG score & & & & 0.767 \\
\hline Tumor location & & & & 0.515 \\
\hline Cervical & 4 & 3 & 7 & \\
\hline Upper & 5 & 6 & 11 & \\
\hline Middle & 11 & 8 & 19 & \\
\hline Lower & 3 & 1 & 4 & \\
\hline 4 & 11 & 14 & 25 & \\
\hline N stage & & & & 0.164 \\
\hline 0 & 2 & 0 & 2 & \\
\hline 1 & 14 & 8 & 22 & \\
\hline 2 & 7 & 10 & 17 & \\
\hline M stage ${ }^{a}$ & & & & 0.542 \\
\hline 0 & 20 & 15 & 35 & \\
\hline 1 & 3 & 3 & 6 & \\
\hline Clinical stage ${ }^{b}$ & & & & 0.112 \\
\hline$\|$ & 1 & 1 & 2 & \\
\hline III & 11 & 3 & 14 & \\
\hline TL & 1 & 0 & 1 & \\
\hline TP & 10 & 8 & 18 & \\
\hline
\end{tabular}

There were no significant differences between radiosensitive and radioresistant patients regarding the distributions of gender, age, ECOG score, tumor location and clinical stage. ECOG, eastern cooperative oncology group; GTV, gross tumor volume; CTV, clinical target volume; PF, platinum plus fluorouracil; TP, platinum plus taxane; a, M1 means Supraclavicular lymphatic node metastasis; IC, Induction chemotherapy; b, according to the 7th AJCC TNM staging system.

increase in proliferation inhibition, indicating that for Eca109 cells, upregulated FAM201A expression likely resulted in cell radioresistance to $\mathrm{X}$-rays (Figure 3E; Supplementary File 4).

In Eca109R cells, when compared with the control cells, siFAM201A exhibited a significant inhibition of cell proliferation, while FAM201A-mimic did not exhibit the increased cell proliferation that was observed in ECA109 cells, indicating that the expression level of FAM201A in Eca109R cells was already at a high level, and thus, further elevation of FAM201A expression was not possible to enhance its radioresistance. These results indicated that, whether in cases of intrinsic or acquired radioresistance, si-FAM201A may enhance ESCC cell radiosensitivity, which may therefore be a novel effective target strategy for sensitizing ESCC to radiotherapy (Figure 3F; Supplementary File 4).

\section{FAM201A Knockdown Enhanced the Radiosensitivity of ESCC in vivo}

To confirm the efficacy of si-FAM201A on radiosensitivity in vivo, a xenograft tumor mouse model was established. A total of 15 mice with similar weights and dates of birth were selected in the present study (male: female $=8: 7$ ). When compared with the control groups, FAM201A knockdown (shFAM201A) significantly blocked tumor growth (decreased tumor volume and weight), suggesting that the silenced FAM201A expression enhanced radiosensitivity, thereby confirming that 


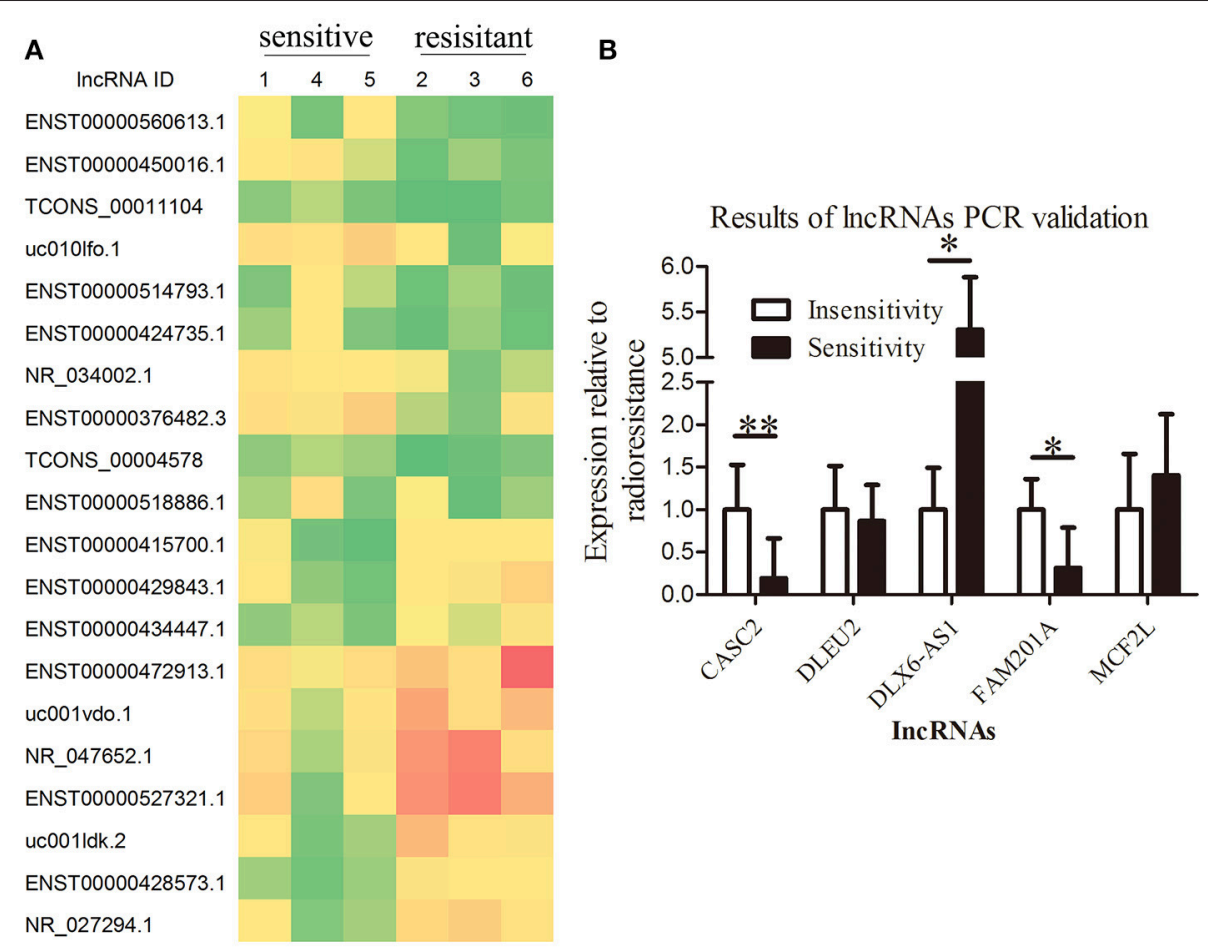

FIGURE 1 | Overexpression of IncRNA FAM201A is highly correlated with the radiosensitivity of ESCC and is associated with poor survival. (A) A heatmap presenting the gene expression levels in RNA samples isolated from three radiosensitive and three radioresistant ESCC tumor tissues by microarray assays. (B) Differential expression of the potential IncRNAs related to radiosensitivity (CASC2, FAM201A, DLEU2, DLX6-AS1, and MCF2L-AS1) in radiosensitive ( $n=20$ ) and radioresistant $(n=15)$ ESCC tumor tissues by reverse transcription-quantitative polymerase chain reaction. ${ }^{*} P<0.05,{ }^{* *} P<0.01$.
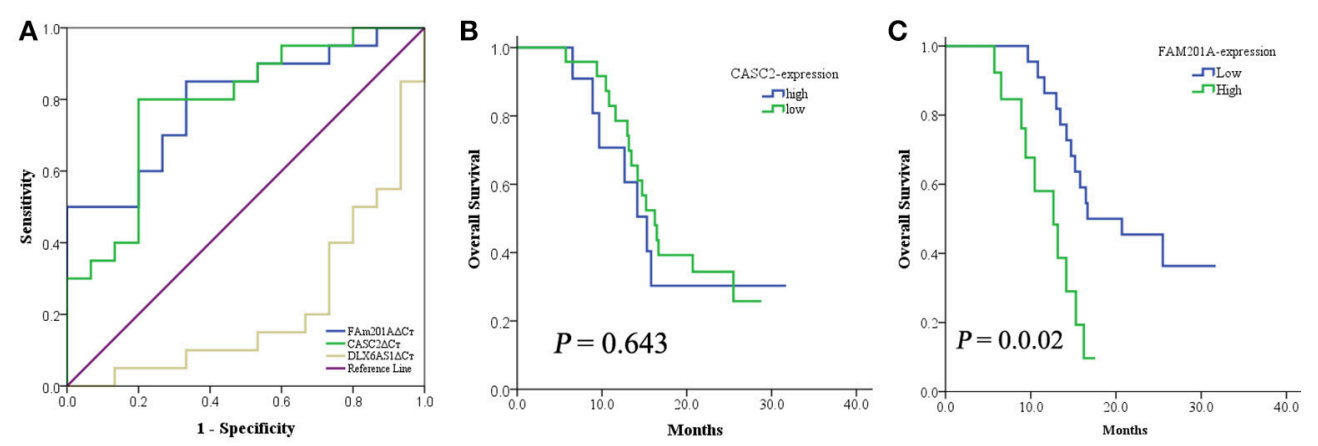

FIGURE 2 | (A) The ROC curve of IncRNA CASC2, FAM201A, and DLX6-AS1. When compared with the IncRNA DLX6-AS1, FAM201A, and CASC2 yielded a superior AUC with specificity and sensitivity for distinguishing radiosensitive ESCC tumor tissues from radioresistant ESCC tumor tissues. (B) The 1-year OS rate between patients with low- $(n=24)$ and high-expression $(n=11)$ of CASC2, was not different. (C) The 1-year OS rate between patients with low- $(n=22)$ and high-expression $(n=13)$ of FAM201A was significantly different $(P=0.001)$.

FAM201A could induce radiosensitivity in vivo (Figures 3G,H; Supplementary File 4).

\section{FAM201A Negatively Regulated the Expression of miR-101}

Using Starbase 2.0, miR-101 and miR-590 were predicted to have complementary base pairings with FAM201A. Accordingly, luciferase reporter vectors containing the Wt or a Mut FAM201A binding site were established and co-transfected with miR101 into Eca109 cells. The same process was performed for miR-590.

The results demonstrated that the ectopic expression of miR-101 was markedly suppressed by co-transfection with the FAM201A mutant sequence in the Eca109 cell luciferase activity reporter assay. However, neither pGL3-FAM201A-Wt reporter nor pGL3-FAM201A-Mut transfection in Eca109 cells affected miR-590 expression (Figures 4A,B; Supplementary File 5). 
TABLE 3 | Treatment results in the high and low FAM201A expression groups.

\begin{tabular}{|c|c|c|c|c|}
\hline Variable & $\begin{array}{l}\text { Low FAM201A } \\
\text { expression, } n\end{array}$ & $\begin{array}{l}\text { High FAM201A } \\
\text { expression, } n\end{array}$ & Total $n$ & $P$-value \\
\hline T stage & & & & 0.161 \\
\hline 2 & 0 & 2 & 2 & \\
\hline 3 & 11 & 5 & 16 & \\
\hline 4 & 11 & 6 & 17 & \\
\hline N stage & & & & 0.998 \\
\hline 0 & 3 & 2 & 5 & \\
\hline 1 & 10 & 6 & 16 & \\
\hline 2 & 7 & 4 & 11 & \\
\hline 3 & 2 & 1 & 3 & \\
\hline M stage & & & & 0.388 \\
\hline 0 & 20 & 13 & 33 & \\
\hline 1 & 2 & 0 & 2 & \\
\hline Tumor response, $n(\%)$ & & & & 0.001 \\
\hline $\mathrm{CR}$ & 1 & 0 & 1 & \\
\hline PR & 17 & 2 & 19 & \\
\hline SD & 3 & 6 & 9 & \\
\hline PD & 1 & 5 & 6 & \\
\hline Pattern of failure, $n$ & & & & 0.177 \\
\hline Locoregional alone & 9 & 4 & 13 & \\
\hline $\begin{array}{l}\text { Locoregional and } \\
\text { distant }\end{array}$ & 0 & 2 & 2 & \\
\hline Distant alone & 4 & 4 & 8 & \\
\hline $\begin{array}{l}1 \text {-year overall survival } \\
\text { rate }(\%)\end{array}$ & 45.5 & 9.7 & & 0.002 \\
\hline
\end{tabular}

The level expression of FAM201A was not correlated with the tumor stage, whatever in term of T stage or N stage. Compared with low expression FAM201A, patients with high expression of FAM201A resulted in poorer short-term response to RT. CR, complete response; $P R$, partial response; $S D$, stable disease; $P D$, progressive disease.

\section{FAM201A Upregulated ATM and mTOR Expression by Acting as a miR-101 Sponge}

To further evaluate the regulatory relationship between FAM201A and miR-101, Eca109 cells were transfected with si-FAM201A and FAM201A-mimic sequences and matched controls. The results revealed that miR-101 expression was significantly downregulated in FAM201A-mimic Eca109/Eca109R cells, and was notably upregulated in siFAM201A-transfected Eca109/Eca109R cells (Figures 4C,D). Taken together, these results indicated that FAM201A suppressed the expression of miR-101 (Supplementary File 6).

Using TargetScan, ATM and mTOR were predicted to be the downstream targets of miR-101. In Eca109/Eca109R cells, the expression of ATM and mTOR was increased while that of miR101 was decreased in FAM201A-mimic cells when compared with control cells. When FAM201A expression was decreased, the expression of ATM and MTOR was downregulated while that of miR-101 was increased. Compared with non-irradiated cells, the expression of ATM and mTOR increased after Xray irradiation. Western blotting confirmed the results of PCR (Figure 5; Supplementary File 6).

\section{DISCUSSION}

The earliest study on lncRNAs associated with radiosensitivity in ESCC was reported by Tong et al. in 2014 (Tong et al., 2014). In this study, they revealed that, when compared with normal paracarcinoma tissue, tumor tissues with a low expression of IncRNA LOC285194 exhibited a larger tumor size, poorer histological grade, had an advanced TNM stage, more lymph node and distant metastases, and was significantly negatively correlated with the pathological response to RT than the LOC285194-high group. Subsequently, researchers have revealed another three IncRNAs related to ESCC radiosensitivity, including BOKAS (Zhang et al., 2015), MALAT1 (Li et al., 2016), and AFAP1AS1 (Zhou et al., 2016). However, clinical trials for evaluating such lncRNAs related to ESCC radiosensitivity are lacking as the mechanism for how lncRNAs regulate radiosensitivity has yet to be fully elucidated, and so no promising lncRNAs have been applied in the clinic.

In the present study, we identified that the IncRNA FAM201A contributed the most to the radioresistance of ESCC regardless of the tumor stage. The FAM201A gene is a $2.9 \mathrm{Kbp}$ long gene located in genomic 9p13.1 (Humphray et al., 2004) that results in RNA transcripts without ORFs, which means that it has no protein-coding potential. FAM201A in human diseases has been reported crudely in Obsessive-compulsive disorder and Tourette's syndrome by Yu et al. (2015), while it was first mentioned in cancer (colorectal) by Matsumura et al. (2017). Recently, Huang et al. revealed that the biofunction of FAM201A was involved in the development of Osteonecrosis of the femoral head (Huang et al., 2018). However, the molecular mechanism of IncRNA FAM201A function has not been studied. To the best of our knowledge, the present study was the first to report on the correlation of FAM201A with ESCC radiosensitivity and to investigate its potential molecular mechanism, in order to elucidate whether it may be a biomarker for the prognosis and prediction of the patient's response to RT.

The results revealed that patients with FAM201A overexpression had poorer radiosensitivity and inferior survival. Conversely, lower FAM201A expression in ESCC was associated with improved radiosensitivity and a good prognosis, indicating that lnc-FAM201A may serve as a predictor of radiosensitivity in ESCC.

Subsequently, we performed experiments in vitro and in vivo to confirm the functions of FAM201A. In vitro, the overexpression of FAM201A was demonstrated to promote Eca109 cell proliferation; while decreasing FAM201A expression inhibited cell proliferation. The difference in radioresistance following the overexpression of FAM201A in Eca109 and Eca109R cells indicated that FAM201A upregulation likely resulted in cell radioresistance to X-rays irradiation. In addition, the similar levels of radiosensitivity following the reduction in FAM201A expression in Eca109 and Eca109R cells suggested that si-FAM201A may enhance the radiosensitivity of both intrinsically and acquired-radioresistant tumor cells, indicating that siFAM201A may serve as an effective sensitizing molecular strategy for ESCC. In vivo, when compared with control groups, FAM201A knockdown significantly blocked xenograft tumor 


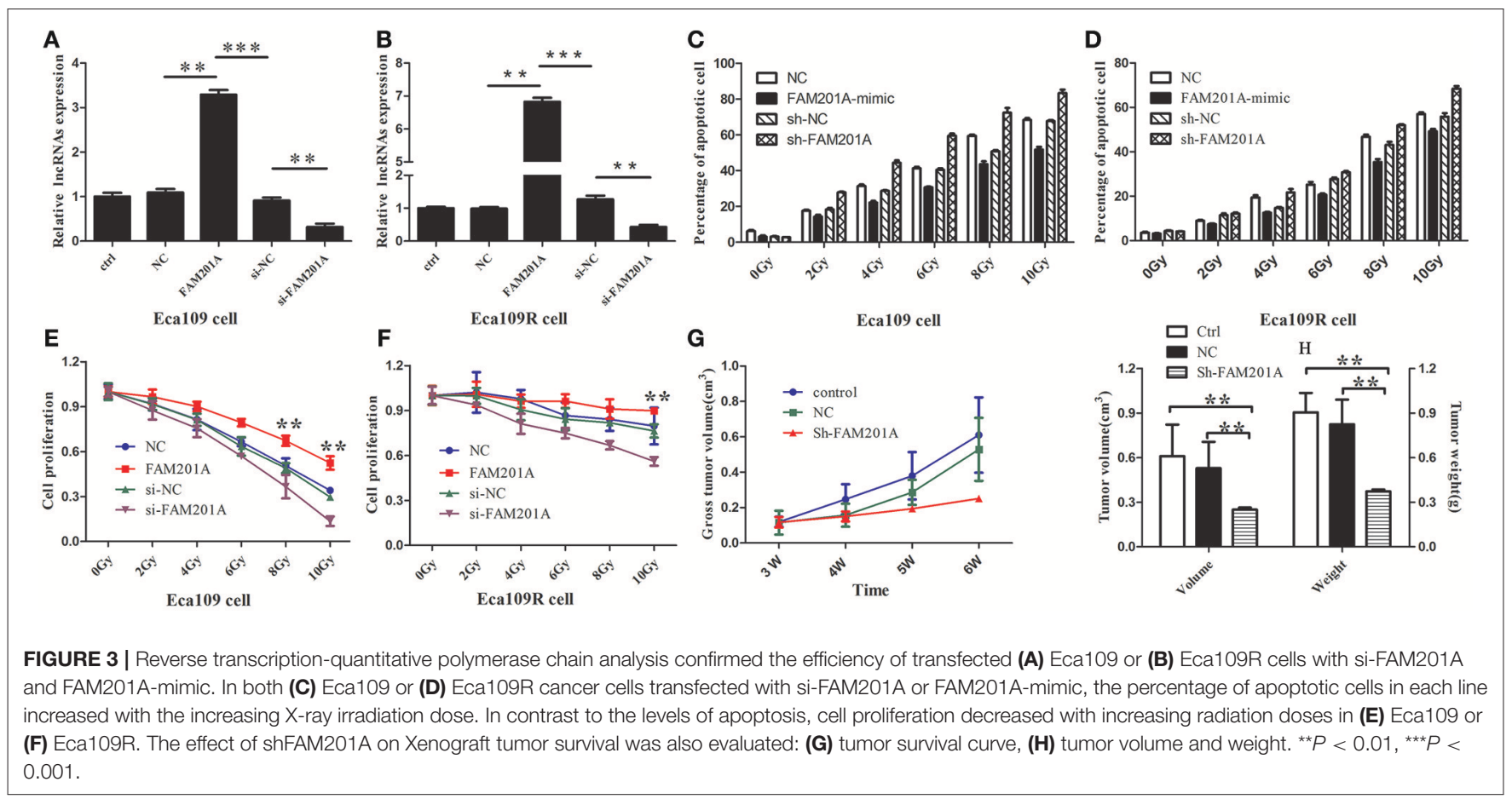

A $\begin{array}{cc}\text { miR-101 } & \begin{array}{c}\text { 3'-aaGTCAATAGTGTCATGACAt-5' } \\ \text { IIIIII }\end{array} \\ \text { Fam201A } & \text { 5'-gtCAATAATAATGTACTGTa-3' } \\ \text { Fam201A mut } & \text { 5'-gtCAATAATAATCATGACAa-3' }\end{array}$

B

miR-590 3'-gacgTGAAAATACTTATTCGAg-5'

|ll||l|

Fam201A 5'-gcaaACATCTTTTCATAAGCTt-3'

Fam201A mut $\quad$ 5'-gcaaACATCTTTTCTATTCGAt-3'
C

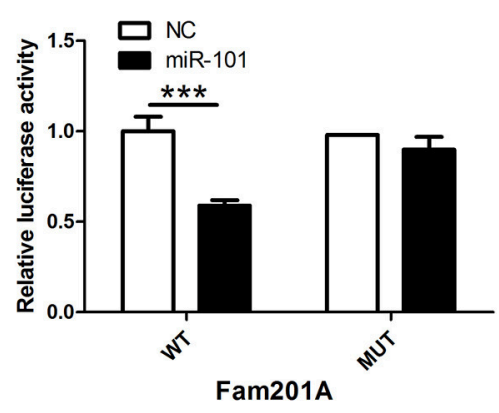

D

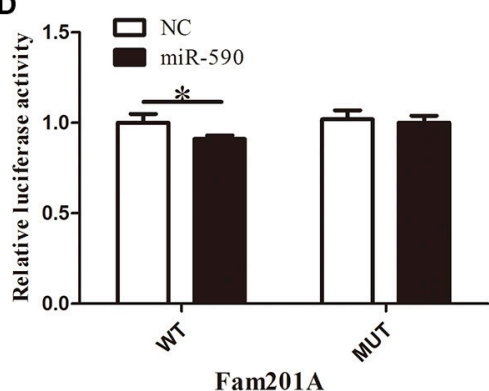

E

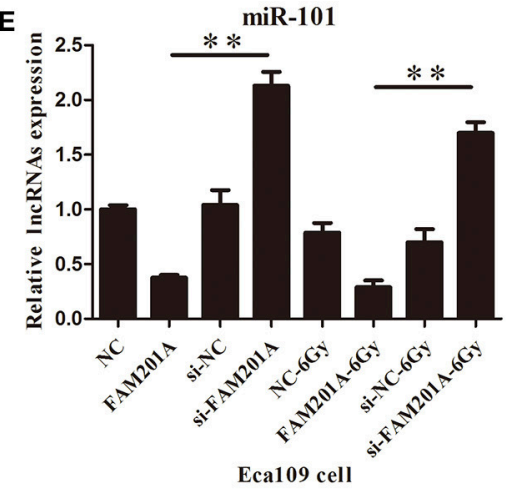

$\mathbf{F}$

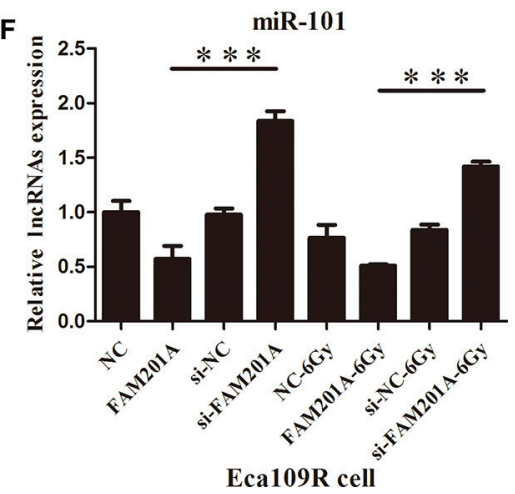

FIGURE 4 | (A) miR-101 and (B) miR-509 were predicted to have complementary base pairings with FAM201A. The relative luciferase activity of the wild-type and mutated FAM201A were compared between (C) miR-101 and (D) miR-590. miR-101 expression was negatively regulated by FAM201A in (E) Eca109 and Eca1 (F) 109R cells. ${ }^{\star} P<0.05,{ }^{\star \star} P<0.01,{ }^{\star \star \star} P<0.001$. 

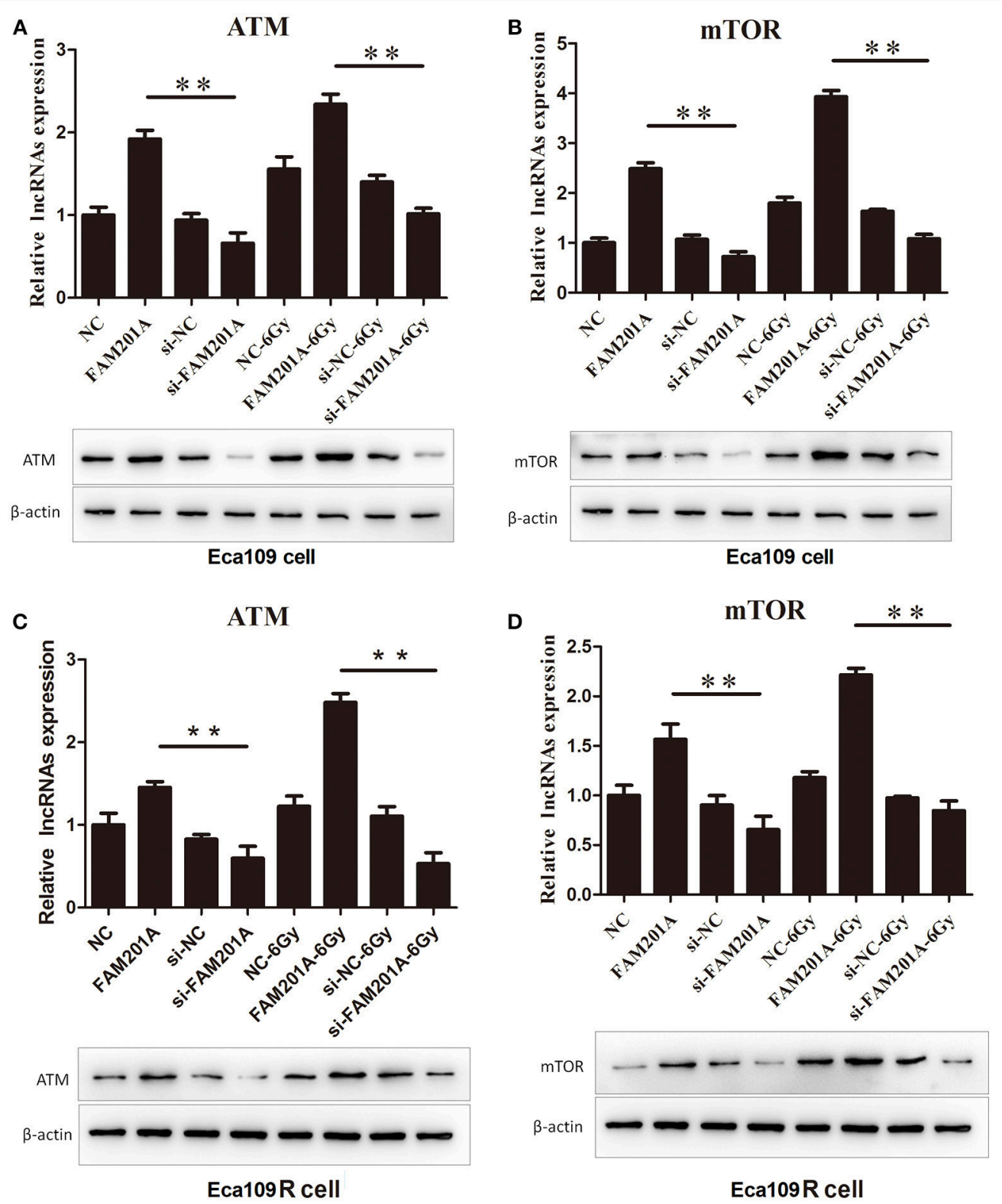

FIGURE 5 | Effects of overexpressed- and si-FAM201A on the expression of miR-101, ATM and mTOR in (A, B) Eca109 and (C, D) Eca109R cells before and after $\mathrm{X}$-ray irradiation. Western blotting validation of ATM and mTOR in Eca109 and Eca109R cells. ${ }^{* \star} P<0.01$.

growth (decreased tumor volume and weight), which confirmed that siFAM201A was able enhance radiosensitivity.

Recently, a competing endogenous RNAs hypothesis proposed that lncRNAs may exert their biological function by acting as a molecular sponge for miRNAs, in turn leading to derepression of miRNA targets (Tay et al., 2014). To explore the molecular mechanism of FAM201A-modulated radiosensitivity in ESCC, we used the online software Starbase 2.0 to predict the downstream target genes, and found that miR-101 and miR-590 had complementary base pairings with FAM201A. Only miR-101, and not miR-590, was observed to directly interact with FAM201A, as determined by the luciferase reporter assay. The qPCR analysis further demonstrated that FAM201A overexpression downregulated miR-101 expression while siFAM201A transfection upregulated miR-101. These results suggested that FAM201A may modulate target gene expression by serving as a "sponge" for miR-101 (Kung et al., 2013).

Further, the role of miRNAs usually depends on what genes they target. The TargetScan analysis showed that ATM and mTOR were the targets of miR-101. Furthermore, qPCR revealed that overexpression of FAM201A leads to the downregulation of miR-101, the upregulation of ATM and mTOR, and resulted in radioresistance; however, depletion of FAM201A led to the upregulation of miR-101, downregulation of ATM, and mTOR, and resulted in radiosensitivity. Additionally, western blotting confirmed these PCR results. 
ATM is the major repair protein involved in the homologous recombination repair (HRR) of ionizing radiation-induced double-strand breaks (RI-DSB). ATM deficiency leads to HRR disorders, increased apoptosis and radiosensitivity (Cliby et al., 1998; Cuddihy and Bristow, 2004; Hammond and Muschel, 2014). Therefore, we hypothesize that FAM201A may regulate ESCC radiosensitivity via a "FAM201A-miRNA101-ATM-HRR" axis.

HRR occurs only in the $S$ and G2 phases of DNA replication, due to the requirement of homologous sister chromatids as a template (Pâques and Haber, 1999). DSBs during the absence of homologous sequence chromosomes requires non-homologous end joining (NHEJ) to achieve DNA repair, which is a repair function performed throughout the cell cycle and was initially considered to be the primary mechanism of RI-DSB repair (Branzei and Foiani, 2008; Beucher et al., 2009). Yan et al. (2010) reported that miR101 regulates the radiosensitivity of cells by regulating the DNA-dependent protein kinase catalytic subunit, an important member of the NHEJ machinery, via mTOR. Therefore, we hypothesize that IncRNA-FAM201A may also modulate cell ionizing radiosensitivity via a "FAM201A-miR-101-mTORNHEJ" axis. In future research, we will focus on the upstream mechanism underlying FAM201A upregulation in regulating ESCC radiosensitivity.

\section{CONCLUSIONS}

In conclusion, the present study revealed that lncRNA FAM201A may be a potential biomarker for predicting radiosensitivity and prognosis, as well as a therapeutic target for enhancing cancer radiosensitivity in ESCC. FAM201A contributed to radioresistance through a FAM201A-miR-101-ATM/mTOR regulatory network in ESCC. However, the upstream mechanism for FAM201A upregulation in regulating ESCC radiosensitivity requires further study.

\section{ETHICS STATEMENT}

This study was subject to approval by the Fujian Medical University Union Hospital Institutional Review Board (No. 2014KY001). All patients signed an informed consent

\section{REFERENCES}

Beucher, A., Birraux, J., Tchouandong, L., Barton, O., Shibata, A., Conrad, S., et al. (2009). ATM and artemis promote homologous recombination of radiation-induced DNA double-strand breaks in G2. EMBO J. 28, 3413-3427. doi: 10.1038/emboj.2009.276

Branzei, D., and Foiani, M. (2008). Regulation of DNA repair throughout the cell cycle. Nat. Rev. Mol. Cell Biol. 9, 297-308. doi: 10.1038/nrm2351

Bray, F., Ferlay, J., Soerjomataram, I., Siegel, R. L., Torre, L. A., and Jemal, A. (2018). Global cancer statistics 2018: GLOBOCAN estimates of incidence and mortality worldwide for 36 cancers in 185 countries. CA Cancer J. Clin. 68, 394-424 doi: 10.3322/caac. 21492

Chaachouay, H., Ohneseit, P., Toulany, M., Kehlbach, R., Multhoff, G., Rodemann, H. P. (2011). Autophagy contributes to resistance of tumor cells to prior to treatment, and all information was anonymized and deidentified prior to its analysis.

\section{AUTHORS CONTRIBUTIONS}

MC, PL, YT, JC, and JL conceived the study, manuscript, and statics analysis. YL, XiaL, MS, XiqL, and AL assistance with collecting clinical data. RY, WN, XZ, YC, and LZ provided assistance with study design and revisions of the manuscript. All authors read and approved the final manuscript.

\section{FUNDING}

This study was supported in part by grants from the Fujian Provincial Health \& Family Planning Commission (Project Number: 2016-ZQN-32), the Fujian Provincial Department of Science \& Technology (Project Number: 2018J01306), the Fujian Provincial Department of Science \& Technology (Project Number: 2017Y9079), the Fujian Provincial Platform for Medical Laboratory Research, and Key Laboratory for Tumor Individualized Active Immunity (Project Number: FYKFKT2017015).

\section{ACKNOWLEDGMENTS}

The authors thank all patients who participated in the present study.

\section{SUPPLEMENTARY MATERIAL}

The Supplementary Material for this article can be found online at: https://www.frontiersin.org/articles/10.3389/fgene. 2018.00611/full\#supplementary-material

File S1 | Results of IncRNA chip microarray.

File S2 | Results of PCR of candidate IncRNAs.

File S3 | FAM201A as a novel IncRNA with a potential function in radiosensitivity.

File S4 | Validation of FAM201A in vitro and in vivo.

File S5 | The results of the luciferase assay.

File S6 | Overexpression of FAM201A and its effect on miR-101/ATM/mTOR in Eca109 and Eca109R cells.

ionizing radiation. Radiother Oncol. 99, 287-292. doi: 10.1016/j.radonc.2011. 06.002

Chen, M. Q., Lin, Q. L., Chen, Y. G., Guo, J. H., Xu, B. H., and Tian, Y. (2017). Neoadjuvant chemotherapy may not benefit esophageal squamous cell carcinoma patients treated with definitive chemoradiotherapy. J. Chin. Med. Assoc. 80, 636-643. doi: 10.1016/j.jcma.2017. 06.014

Chen, X., Liao, R., Li, D., and Sun, J. (2017). Induced cancer stem cells generated by radiochemotherapy and their therapeutic implications. Oncotarget 8 , 17301-17312. doi: 10.18632/oncotarget.14230

Cliby, W. A., Roberts, C. J., Cimprich, K. A., Stringer, C. M., Lamb, J. R., Schreiber, S. L., et al. (1998). Overexpression of a kinase-inactive ATR protein causes sensitivity to DNA-damaging agents and defects in cell cycle checkpoints. EMBO J. 17, 159-169. doi: 10.1093/emboj/17.1.159 
Cooper, J. S., Guo, M. D., Herskovic, A., Macdonald, J. S., Martenson, J. A. Jr, Al-Sarraf, M., et al. (1999). Chemoradiotherapy of locally advanced esophageal cancer: long-term follow-up of a prospective randomized trial (RTOG 85-01). radiation therapy oncology group. JAMA 281, 1623-1627. doi: 10.1001/jama.281.17.1623

Cuddihy, A. R., and Bristow, R. G. (2004). The p53 protein family and radiation sensitivity: yes or no? Cancer Metastasis Rev. 23, 237-257. doi: 10.1023/B:CANC.0000031764.81141.e4

Da, C., Wu, L., Liu, Y., Wang, R., and Li, R. (2017). Effects of irradiation on radioresistance, HOTAIR and epithelial-mesenchymal transition/cancer stem cell marker expression in esophageal squamous cell carcinoma. Oncol. Lett. 13, 2751-2757. doi: 10.3892/ol.2017.5774

Dumont, F. J., and Bischoff, P. (2012). Disrupting the mTOR signaling network as a potential strategy for the enhancement of cancer radiotherapy. Curr. Cancer Drug Targets. 12, 899-924. doi: 10.2174/156800912803251243

Fluss, R., Faraggi, D., and Reiser, B. (2005). Estimation of the Youden index and its associated cutoff point. Biom. J. 47, 458-472. doi: 10.1002/bimj.200410135

Francescone, R. A., Scully, S., Faibish, M., Taylor, S. L., Oh, D., Moral, L., et al. (2011). Role of YKL-40 in the angiogenesis, radioresistance, and progression of glioblastoma. J. Biol. Chem. 286, 15332-15343. doi: 10.1074/jbc.M110.212514

Gwynne, S., Hurt, C., Evans, M., Holden, C., Vout, L., and Crosby, T. (2011). Definitive chemoradiation for oesophageal cancer-a standard of care in patients with non-metastatic oesophageal cancer. Clin. Oncol. (R. Coll. Radiol) 23, 182-188. doi: 10.1016/j.clon.2010.12.001

Hammond, E. M., and Muschel, R. J. (2014). Radiation and ATM inhibition: the heart of the matter. J. Clin. Invest. 124, 3289-3291. doi: 10.1172/JCI77195

Huang, G., Zhao, G., Xia, J., Wei, Y., Chen, F., Chen, J., et al. (2018). FGF2 and FAM201A affect the development of osteonecrosis of the femoral head after femoral neck fracture. Gene 652, 39-47. doi: 10.1016/j.gene.2018.01.090

Humphray, S. J., Oliver, K., Hunt, A. R., Plumb, R. W., Loveland, J. E., Howe, K. L., et al. (2004). DNA sequence and analysis of human chromosome 9. Nature 429, 369-374. doi: 10.1038/nature,02465

Japan Esophageal Society (2017). Japanese classification of esophageal cancer, 11th edition: part II and III. Esophagus 14, 37-65. doi: 10.1007/s10388-016-0556-2

Kung, J. T., Colognori, D., and Lee, J. T. (2013). Long noncoding RNAs: past, present, and future. Genetics 193, 651-669. doi: 10.1534/genetics.112.146704

Li, Z., Zhou, Y., Tu, B., Bu, Y., Liu, A., and Kong, J. (2016). Long noncoding RNA MALAT1 affects the efficacy of radiotherapy for esophageal squamous cell carcinoma by regulating Cks1 expression. J. Oral Pathol. Med. 46, 583-590. doi: $10.1111 /$ jop. 12538

Livak, K. J., and Schmittgen, T. D. (2001). Analysis of relative gene expression data using real-time quantitative PCR and the 2(-Delta delta C(T)) method. Methods 25, 402-408. doi: 10.1006/meth.2001.1262

Lloyd, S., and Chang, B. W. (2014). Current strategies in chemoradiation for esophageal cancer. J. Gastrointest. Oncol. 5, 156-165. doi: 10.3978/j.issn.2078-6891.2014.033

Matsumura, K., Kawasaki, Y., Miyamoto, M., Kamoshida, Y., Nakamura, J., Negishi, L., et al. (2017). The novel G-quadruplex-containing long non-coding RNA GSEC antagonizes DHX36 and modulates colon cancer cell migration. Oncogene 36, 1191-1199. doi: 10.1038/onc.2016.282

Moncharmont, C., Levy, A., Gilormini, M., Bertrand, G., Chargari, C., Alphonse, G., et al. (2012). Targeting a cornerstone of radiation resistance: cancer stem Cell 322, 139-147. doi: 10.1016/j.canlet.2012.03.024

Pâques, F., and Haber, J. E. (1999). Multiple pathways of recombination induced by double-strand breaks in Saccharomyces cerevisiae. Microbiol. Mol. Biol. Rev. 63, 349-404.

Pennathur, A., Gibson, M. K., Jobe, B. A., and Luketich, J. D. (2013). Oesophageal carcinoma. Lancet 381, 400-412. doi: 10.1016/S0140-6736(12)60643-6
Qi, P., and Du, X. (2013). The long non-coding RNAs, a new cancer diagnostic and therapeutic gold mine. Mod. Pathol. 26, 155-165. doi: $10.1038 /$ modpathol.2012.160

Rustgi, A. K., and El-Serag, H. B. (2014). Esophageal carcinoma. N. Engl. J. Med. 371, 2499-2509. doi: 10.1056/NEJMra1314530

Sasaki, Y., and Kato, K. (2016). Chemoradiotherapy for esophageal squamous cell cancer. JPN. J. Clin. Oncol. 46:805. doi: 10.1093/jjco/hyw082

Short, M. W., Burgers, K. G., and Fry, V. T. (2017). Esophageal Cancer. Am. Fam. Physician 95, 22-28.

Spizzo, R., Almeida, M. I., Colombatti, A., and Calin, G. A. (2012). Long noncoding RNAs and cancer: a new frontier of translational research? Oncogene 31:4577. doi: 10.1038/onc.2011.621

Tay, Y., Rinn, J., and Pandolfi, P. P. (2014). The multilayered complexity of ceRNA crosstalk and competition. Nature 505, 344-352. doi: 10.1038/nature12986

Tong, Y. S., Zhou, X. L., Wang, X. W., Wu, Q. Q., Yang, T. X., Lv, J., et al. (2014). Association of decreased expression of long non-coding RNA LOC285194 with chemoradiotherapy resistance and poor prognosis in esophageal squamous cell carcinoma. J. Transl. Med. 12:233. doi: 10.1186/s12967-014-0233-y

Versteijne, E., Van Laarhoven, H. W., van Hooft, J. E., Van Os, R. M., Geijsen, E. D., Van Berge Henegouwen, M. I., et al. (2014). Definitive chemoradiation for patients with inoperable and/or unresectable esophageal cancer: locoregional recurrence pattern. Dis. Esophaqus. 28, 453-459. doi: 10.1111/dote. 12215

Yan, D., Ng, W. L., Zhang, X., Wang, P., Zhang, Z., Mo, Y. Y., et al. (2010). Targeting DNA-PKcs and ATM with miR-101 sensitizes tumors to radiation. PLoS ONE 5:e11397. doi: 10.1371/journal.pone.0011397

Yu, D., Mathews, C. A., Scharf, J. M., Neale, B. M., Davis, L. K., Gamazon, E. R., et al. (2015). Cross-disorder genome-wide analyses suggest a complex genetic relationship between tourette's syndrome and OCD. Am. J. Psychiatry 172, 82-93. doi: 10.1176/appi.ajp.2014

Yu, Z. Q., Zhang, C., Lao, X. Y., Wang, H., Gao, X. H., Cao, G. W., et al. (2012). Long non-coding RNA influences radiosensitivity of colorectal carcinoma cell lines by regulating cyclin D1 expression. Zhonghua Wei Chang Wai Ke Za Zhi 15, 288-291.

Zafar, F., Seidler, S. B., Kronenberg, A., Schild, D., and Wiese, C. (2010). Homologous recombination contributes to the repair of DNA doublestrand breaks induced by high-energy iron ions. Radiat. Res. 173, 27-39. doi: $10.1667 / R R 1910.1$

Zhang, H., Luo, H., Hu, Z., Peng, J., Jiang, Z., Song, T., et al. (2015). Targeting WISP1 to sensitize esophageal squamous cell carcinoma to irradiation. Oncotarget 6:6218. doi: 10.18632/oncotarget.3358

Zhou, X. L., Wang, W. W., Zhu, W. G., Yu, C. H., Tao, G. Z., Wu, Q. Q., et al. (2016). High expression of long non-coding RNA AFAP1-AS1 predicts chemoradioresistance and poor prognosis in patients with esophageal squamous cell carcinoma treated with definitive chemoradiotherapy. Mol. Carcinog. 55, 2095-2105. doi: 10.1002/mc.22454

Conflict of Interest Statement: The authors declare that the research was conducted in the absence of any commercial or financial relationships that could be construed as a potential conflict of interest.

Copyright (c) 2018 Chen, Liu, Chen, Chen, Shen, Liu, Li, Li, Lin, Yang, Ni, Zhou, Zhang, Tian, Li and Chen. This is an open-access article distributed under the terms of the Creative Commons Attribution License (CC BY). The use, distribution or reproduction in other forums is permitted, provided the original author(s) and the copyright owner(s) are credited and that the original publication in this journal is cited, in accordance with accepted academic practice. No use, distribution or reproduction is permitted which does not comply with these terms. 